\title{
ANÁLISIS Y COMPARACIÓN DE ISDB-T UTILIZANDO MODULACIONES OFDM Y CC-OFDCM
}

\section{ANALYSIS AND COMPARATION OF ISDB-T USING MODULATIONS OFDM AND CC-OFDCM}

\author{
José Gómez ${ }^{1}$, Holger Lapo ${ }^{1}$, Luis Oñate ${ }^{1, *}$
}

\section{Resumen}

La Televisión Digital Terrestre está orientada a sustituir las transmisiones de televisión analógica, al utilizar la modulación OFDM (multiplexación por división de frecuencias ortogonales), con esta modulación se puede realizar transmisiones para equipos fijos y móviles. Sin embargo, en el caso de sistemas de recepción de televisión digital como teléfonos inteligentes, portátiles y otros dispositivos móviles tienen problemas en la recepción causados por el efecto multicamino. En este proyecto se realiza la simulación de las modulaciones OFDM y CC-OFDM (Multiplexación por División de Frecuencias Ortogonales mediante Codificación por Convolución) para el estándar de televisión ISDB-T (Radiodifusión Digital de Servicios Integrado para Televisión). Por medio de Simulink del entorno Matlab, se simuló los diagramas de bloques de ambas arquitecturas para observar los resultados al transmitir datos, midiendo el BER (Tasa de Bit Errado), el retardo y el número de portadoras, bajo los siguientes canales: AWGN (Ruido Gaussiano Blanco Aditivo), Terreno tipo A (terreno rural) y Terreno tipo C (terreno urbano). De los resultados obtenidos se muestra que la modulación CC-OFDM tiene una mayor eficiencia, debido a que presenta un BER mayor y reducción en el retardo en la recepción.

Palabras clave: CC-OFDM, ISDB-T, multicamino, OFDM, Simulink

\section{Abstract}

Digital Terrestrial Television (DTT) is focused on replacing analogue television transmissions, however, with the modulation used OFDM (orthogonal frequency division multiplexing), transmissions can be made for fixed equipment. However, in the case of digital television reception systems such as smartphones, laptops and other mobile devices have reception problems caused by the multipath effect. In this project, the OFDM and CC-OFDM modulations (Orthogonal Frequency Division Multiplexing by Convolution Coding) are implemented in software Simulink for the ISDB-T (Integrated Services Digital Television Broadcasting) standard. By means of Simulink de Matlab the respective block diagrams of their architectures were implemented to observe the results when transmitting data, measuring the BER (Bit Error Rate), the delay and the number of carriers, under three types of channels such as: AWGN (Additive White Gaussian Noise), Type A land (rural land) and Type $\mathrm{C}$ land (urban land) in order to observe which modulation presents the best efficiency. From the obtained results it is shown that CC-OFDM modulation has a higher efficiency, because it has a higher BER and reduction in the reception delay.

Keywords: CC-OFDM, ISDB-T, Multiway, OFDM, Simulink.

\footnotetext{
$\overline{1, *}$ Carrera de Ingeniería Electrónica, Universidad Politécnica Salesiana, Ecuador. Autor para correspondencia lonate@ups.edu.ec. (D) http://orcid.org/0000-0003-0741-2115, (D) http://orcid.org/0000-0002-4885-4414, (D) http://orcid.org/0000-0002-1323-1858
}

Recibido: 22-10-2018, aprobado tras revisión: 12-12-2018

Forma sugerida de citación: Gómez, J.; Lapo, H. y Oñate, L. (2019). «Análisis y comparación de ISDB-T utilizando modulaciones OFDM y CC-OFDCM». IngEnIUs. N. ${ }^{\circ} 21$, (enero-junio). pp. 71-77. DOI: https://doi.org/10.17163/ings. n21.2019.07. 


\section{Introducción}

La televisión digital está orientada a sustituir las transmisiones de televisión analógica porque tiene la ventaja de incluir aplicaciones interactivas como son las encuestas, la programación y sus horarios, señales de emergencia entre otros, así como diferentes tipos de calidad de la señal en alta definición (HD) y definición estándar (SD) [1].

Cuando se reciben señales de televisión digital en dispositivos como teléfonos inteligentes, portátiles entre otros se tienen problemas de ruido impulsivo causados por la trayectoria multicamino en el estándar ISDB-T al utilizar la modulación habitual OFDM [2].

El estándar ISDB-T (Radiodifusión Digital de Servicios Integrado para Televisión) tuvo sus orígenes en Japón habiendo sido desarrollado en los últimos años de la década de 1990. El objetivo principal de este estándar de televisión digital fue lograr que HDTV (TV de Alta Definición) y la SDTV (TV Digital Estándar) simultáneamente se transmitan. ISDB-T fue diseñado para funcionar en canales con un ancho de banda de 6, 7 y $8 \mathrm{MHz}$ respectivamente. En el Ecuador este estándar debe trabajar con un canal de $6 \mathrm{MHz}$ [3].

En la última década la multeplexación por división de frecuencia (OFDM) se ha constituido en la base de los sistemas de telecomunicaciones y que es una técnica utilizada en los sistemas de trasmisión inalámbrico [4] y en los de fibra óptica [5]. Las ventajas de OFDM son el manejo de interferencia multicamino, la mitigación de la interferencia intersímbolo (ISI) causada por la tasa de bits errados en la frecuencia selectiva en ambientes con desvanecimiento [6] OFDMA es una técnica multiportadora de trasmisión el cual es reconocida como una de las mejores para redes inalámbricas bidireccionales en sistemas de comunicaciones y para trasmisión de televisión digital tipo broadcasting en especial en el sistema de televisión ISDB-t de uso en Ecuador, además, se utiliza en comunicaciones satelitales y espaciales, reduciendo el error en las mismas. En la trasmisión digital y almacenamiento de datos el criterio de calidad utilizado es la tasa de error de bit (BER) comparando el número de bit errados y el número de bits transmitidos.

Las transmisiones de televisión terrestre digital tipo broadcasting tiene a ser reflejada por edificios y montañas; la propagación tiende a convertirse en multicamino. Para lograr evitar la interferencia, la televisión digital ISDB-t que es utilizado en nuestro país se basa en el sistema del Japón con la revisión del Brasil, utiliza OFDM como esquema de modulación. Esta técnica no es robusta y presenta problemas que en transmisiones multicamino y genera grandes retardos los cuales pueden exceder el intervalo de guarda (GI) o picos elevados de la potencia de trasmisión [7]. Si se producen picos en el dominio del tiempo ya que muchas componentes de subportadoras son añadidas al utilizar la operación de la transformada rápida de Fourier inversa (IFFT) [8]. Más allá de esto los sistemas OFDM se caracterizan por tener un alto PAPR (relación entre potencia pico y potencia promedio) con respecto a sistemas con una sola portadora. Al pasar por un elemento no lineal las señales con un alto PAPR OFDM tal es el caso de un amplificador de alta potencia (HPA), [9] provoca una señal que está fuera del rango del espectro de las portadoras que interferirá con los canales adyacentes, y dentro del espectro de las portadoras lo que causa distorsión, atenuación y un nivel de corriente continua (offset) en la señal recibida. El HPA con un rango dinámico grande provoca que las pérdidas en el sistema de comunicación sean altas. Se puede reducir con componentes que tengan bajas pérdidas, pero eso lo vuelve muy costoso. El efecto del desvanecimiento se logra compensar utilizando CCOFDM [10].

La modulación CC-OFDM en el sistema de multiplexación por división de frecuencia ortogonal (OFDM) se transformó en una herramienta muy utilizada en la tecnología actual. De igual manera, que otros sistemas de comunicación, el sistema OFDM requiere usar la codificación de canal para disminuir el BER como el CC-OFDM (Multiplexación por División de Frecuencias Ortogonales mediante Codificación por Convolución) [11]. En la Figura 1 se observa el diagrama de bloques de la modulación CC-OFDM (Multiplexación por División de Frecuencias Ortogonales mediante Codificación por Convolución) [12].

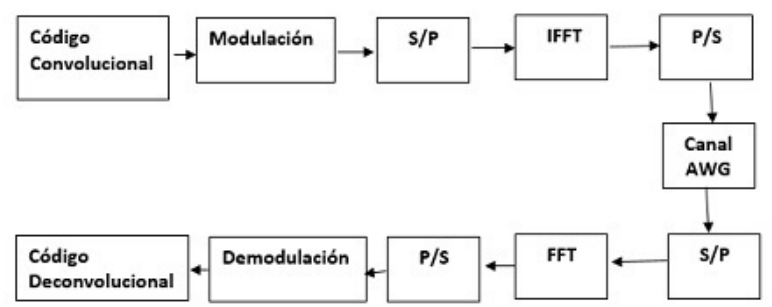

Figura 1. OFDM con el código de convolución.

En el artículo utilizando las arquitecturas ISDB-t se realiza un análisis y simulación para establecer en qué medida mejora la eficiencia en el ambiente multicamino, además de observar parámetros al como el BER, delay y número de portadoras utilizando la modulación CC-OFDM.

El diagrama de bloques que se observa en la Figura 1 está formado por el generador del código de convolución el cual es un tipo de código de corrección de errores, la modulación en este caso es 64 QAM, aunque se puede utilizar otros tipos de modulaciones como QPSK, QAM, 16 QAM, 32 QAM, un conversor serial a paralelo $(\mathrm{P} / \mathrm{S})$, Transformada Rápida de Inversa Fourier (IFFT), canal que es el medio de transmisión por el cual se transmite los datos donde 
se introducirá atenuación, ruido y desvanecimiento de la señal, un conversor Paralelo a Serial (S/P), Transformada Discreta de Fourier (DFT), un segundo conversor $(\mathrm{P} / \mathrm{S})$, demodulación y, finalmente, la decodificación del código convolucional [13].

\section{Materiales y métodos}

En el modelo ISDB-T se debe tener en cuenta los parámetros y valores configurables para el estándar
ISDB-T ya que al seleccionarlos se definirá completamente el modo de operación de los bloques del sistema como se describe en el documento [14]. Se observa en la Figura 2 el diagrama de bloques de transmisión y recepción para el estándar ISDB-T con modulación OFDM elaborado en Simulink de Matlab. Los bloques empleados en la simulación del estándar ISDB-T con modulación OFDM se detallan a continuación:

El generador de imagen emplea el bloque Signal From Worskpace que es el encargado de importar los datos de la imagen desde el Workspace de Matlab [15].

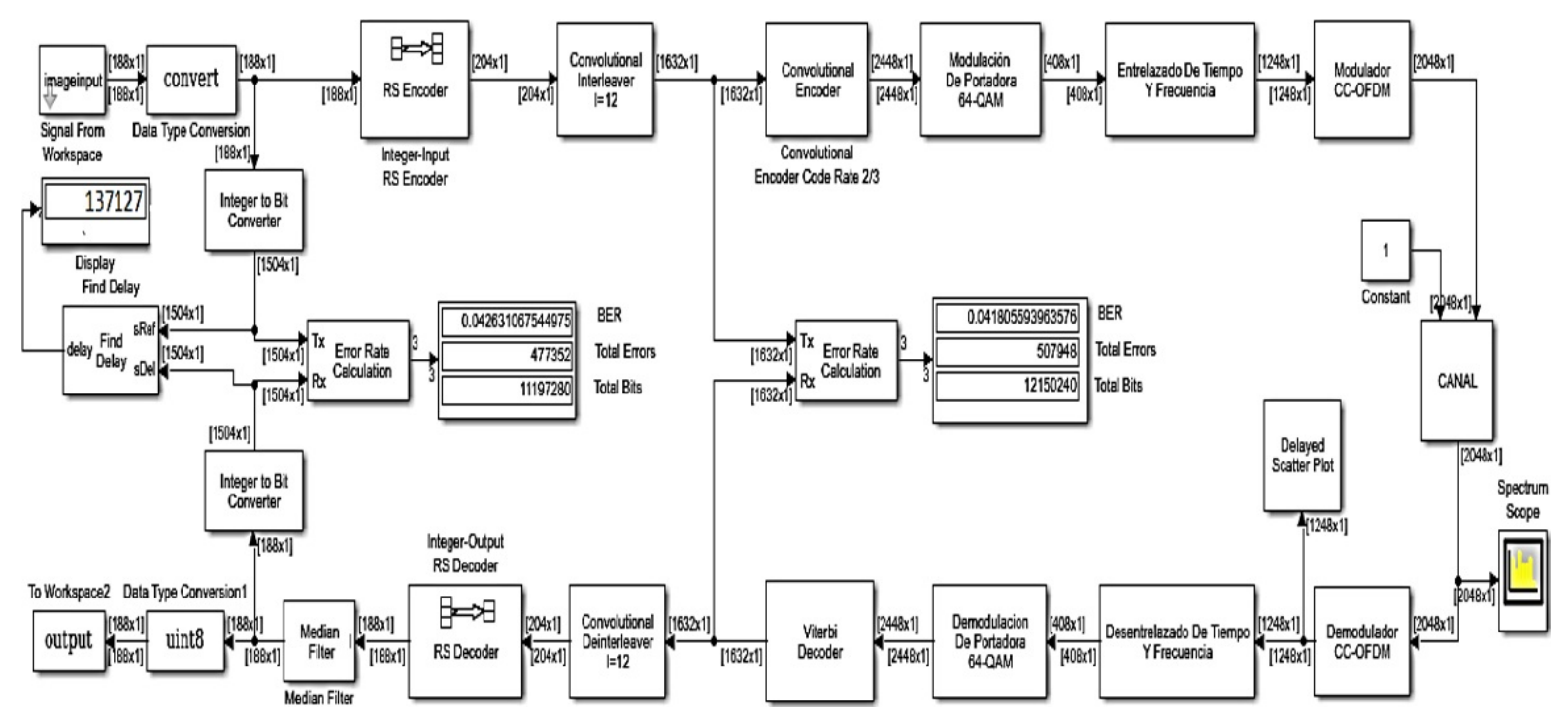

Figura 2. Diagrama de bloques para el estándar ISDB-T con modulación OFDM elaborado en Simulink de Matlab.

El codificador Reed Solomon realiza un proceso de corrección de errores basado en bloques, permitiendo procesar por bloques una cierta cantidad de símbolos de datos. Para este caso emplea un código de la forma $\mathrm{k} / \mathrm{n}=188 / 204[16]$.

El convertidor de enteros a bits establece un valor de 8, para obtener un total 1632 bits valor requerido en la norma [17]. La modulación de portadora emplea un bloque llamado matrix interleaver que hará el proceso de entrelazado de bits. Para posteriormente realizar el proceso de mapping que consta de dos bloques, un conversor de bit a entero y un modulador rectangular QAM, para tener una modulación 64-QAM es necesario colocar el valor de 6 el conversor de bits a enteros, así como también ingresar el valor de 64 en el modulador QAM [18].

El entrelazado de tiempo y frecuencia consta de un buffer, en donde se ingresan el número de filas y columnas indicados por el estándar $(96 \times 204)$, realizan una conversión serial a paralelo. El bloque matrix interleaver contiene las filas y columnas antes mencionadas para poder transmitir correctamente los datos. Finalmente, se tiene un segundo buffer que almacena los 1248 datos que pertenecen al estándar [19].

En la modulación OFDM se asigna el número total de portadoras (2048), luego se ingresar en el parámetro número de bandas de guarda el valor de [400; 400], que permitirá obtener los 1248 datos que usa el simulador.

Se observa en la Figura 3 el diagrama de bloques de transmisión y recepción mencionados previamente para CC-OFDM [20].

En el canal AWGN adicionalmente se implementó una línea de 3 taps que indica el proceso del efecto multicamino denominados canales SUI (Standford University Interim); además, un bloque AWGN Channel que ingresa ruido blanco gaussiano aditivo. Se utiliza un modelo de comunicaciones por radio frecuencia que puede ser utilizado en Wimax, televisión digital, comunicaciones inalámbricas a larga distancia [21]. El demodulador OFDM realiza el proceso inverso de la modulación OFDM. En el desentrelazado de tiempo y frecuencia se realiza un proceso inverso en el cual se debe obtener a la salida un vector de $[272 \times 1]$ que debe ser igual a lo indicado en el proceso de transmisión y pasar a la siguiente etapa [22]. 


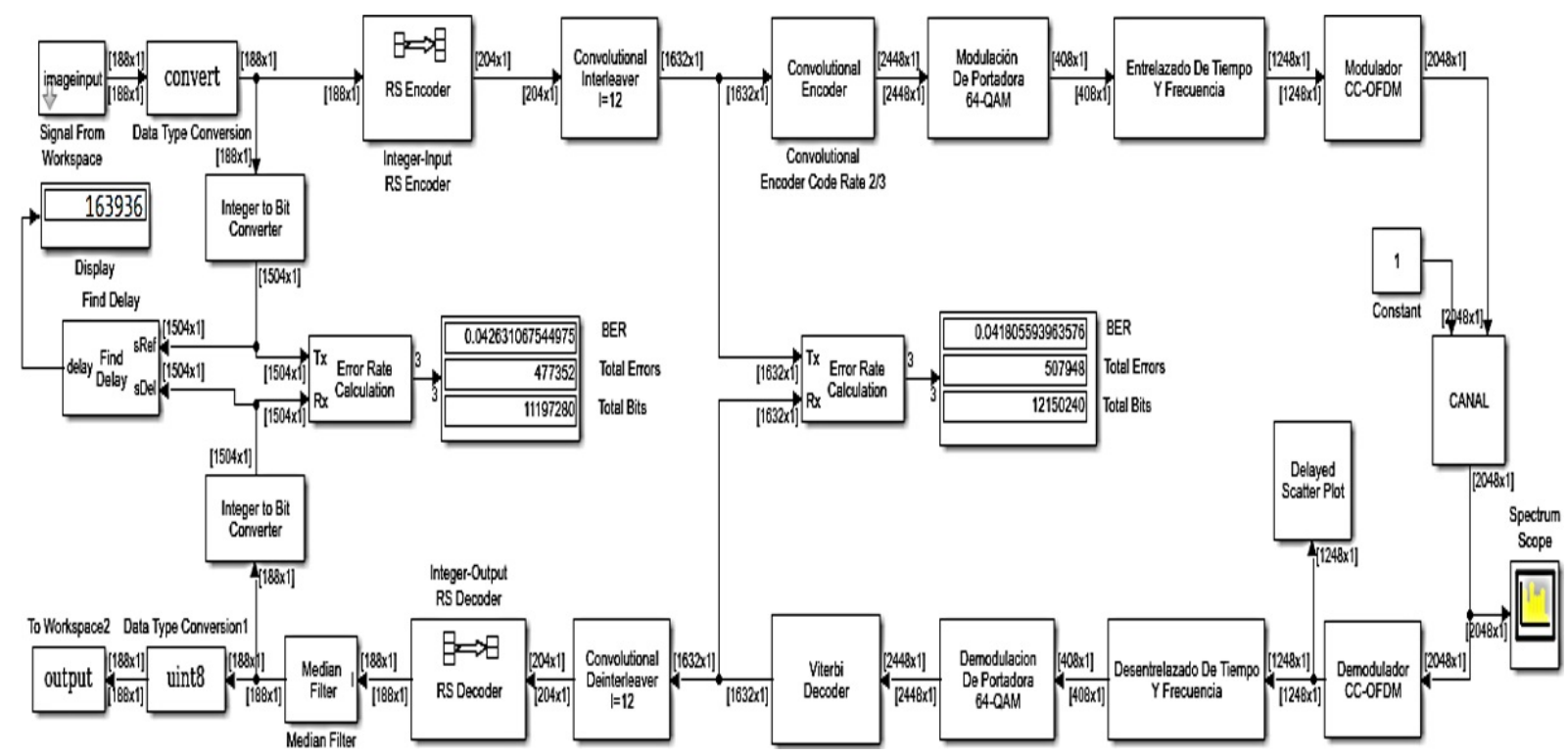

Figura 3. Diagrama de bloques para el estándar ISDB-T con modulación CC-OFDM elaborado en Simulink de Matlab.

La demodulación de portadora en este subsistema se realiza el desentrelazado de bits con el bloque $m a-$ trix deinterleaver para posteriormente demodular a 64-QAM con el bloque demodulador rectangular QAM. El convertidor de bits a enteros convierte los 1632 bits ingresados en el matrix deinterleaver a 204 enteros que se necesita en el siguiente bloque [23]. El decodificador Reed Solomon recupera un vector con mensaje binario, los parámetros deben concordar con los valores de la entrada del codificador Reed Solomon, para obtener los mismos enviados [24]. El filtro mediano emplea un bloque Median Filter que se utilizó para disminuir el efecto de sal y pimienta que presentaban las imágenes recibidas y que se introduce en el canal de comunicaciones, para lo cual se encuentra la media de los pixeles de las imágenes procesadas y aproxima mediante un kernel los valores de los pixeles cercanos disminuyendo el ruido [25]. En la presentación de la imagen transmitida contiene el bloque To Worskpace cuya finalidad es para el estándar ISDB-T con modulación CC-OFDM en Simulink de Matlab.

Los bloques de Simulink con modulación CCOFDM usan para la corrección de errores de entrelazado y desentrelazado de bits. Además, se agrega un codificador convolucional y su inverso un decodificador Viterbi, para obtener una menor tasa de errores [26]. $\mathrm{El}$ entrelazador convolucional contiene el bloque Convolutional Interleaver que ejecuta un entrelazado convolucional de bytes para mejorar el desempeño contra errores. Al codificador convolucional se aplicó un vector de Punzonado eligiendo una tasa de codificación de $2 / 3$ con una extensión de 7 bits de convolución [12]. El bloque Viterbi Decoder es utilizado para decodificar la señal que fue codificada por el codificador convolucional, empleando el algoritmo Viterbi. Este decodificador utiliza las características del Trellis de código convolucional, este algoritmo Viterbi reduce la complicación de los cálculos evitando tomar en cuenta todas las secuencias posibles [27]. El desentrelazador convolucional reordena los símbolos de una señal que fue entrelazada empleando registros de desplazamiento cada uno con retraso fijo.

\section{Resultados y discusión}

Los resultados del BER para los diferentes estándares TDT y sus respectivas modulaciones con una señal a ruido de 19 [dB], ya que este valor permitirá obtener una imagen clara con bajo ruido. Los resultados se pueden apreciar en la siguiente Tabla 1.

Tabla 1. Resultados del Bit Error Rate

\begin{tabular}{ccc}
\hline Estándar & \multicolumn{2}{c}{ ISDB-T (BER) } \\
\hline SNR[dB] & OFDM & CC-OFM \\
19 & 0,04333 & 0,04263 \\
\hline
\end{tabular}

Se realizaron cálculos con una $\mathrm{SNR}=16[\mathrm{~dB}][22]$ ya que este valor permitirá obtener la mejor eficiencia al aplicar efecto multicamino, en el terreno A como en el terreno C.

Para cada una de las modulaciones respectivas de los estándares de TDT estudiados se obtiene la siguiente Tabla 2 que indica el valor del retardo correspondiente en segundos. 
Tabla 2. Resultados del retardo

\begin{tabular}{ccc}
\hline Estándar & \multicolumn{2}{c}{ ISDB-T $($ BER) } \\
\hline Modulaciones & OFDM & CC-OFM \\
Delay & $6,9963[\mathrm{~ms}]$ & $8,3640[\mathrm{~ms}]$ \\
\hline
\end{tabular}

Teniendo como señal de entrada una imagen RGB se obtuvo los diferentes resultados entre BER vs. SNR en el cual se consigue resultados distintos debido a la variación que se produce en la relación señal a ruido. Esta variación se la realiza en los 2 canales SUI (Standford University Interim) implementados en la simulación con el fin de observar cuál de los estándares tiene mejor comportamiento ante este efecto multicamino como se observa en la Tabla 3. Siendo el BER menor en CC-OFDM.

Tabla 3. Resultados del BER vs. SNR $[\mathrm{dB}]$ con efecto multicamino

\begin{tabular}{|c|c|c|c|c|}
\hline \multicolumn{5}{|c|}{ ISDB-T } \\
\hline & \multicolumn{2}{|c|}{ OFDM VER } & \multicolumn{2}{|c|}{ CC-OFM VER } \\
\hline SNR Db & Terreno A & Terreno C & Terreno A & Terreno C \\
\hline 10 & 0,13040 & 0,11866 & 0,32612 & 0,24866 \\
\hline 12 & 0,10997 & 0,09436 & 0,20365 & 0,09556 \\
\hline 14 & 0,09357 & 0,07342 & 0,10164 & 0,04618 \\
\hline 16 & 0,08112 & 0,05699 & 0,05799 & 0,04277 \\
\hline 48 & 0,07194 & 0,04702 & 0,04650 & 0,04263 \\
\hline 20 & 0,06546 & 0,04291 & 0,04439 & 0,04263 \\
\hline
\end{tabular}

En la Figura 4 se muestran los resultados pertinentes para el estándar ISDB-T, donde se observa que tanto la curva del terreno A (urbano) y terreno $\mathrm{C}$ (rural), de color gris y amarillo respectivo tienen para un SNR de $16 \mathrm{~dB}$ tiene una mejor respuesta del VER con la modulación CC-OFDM con respecto a las curvas en los mismos tipos de terrenos con la modulación OFDM. Usando esta modulación en multicamino se logra transmitir imágenes con menor ruido sal y pimienta.

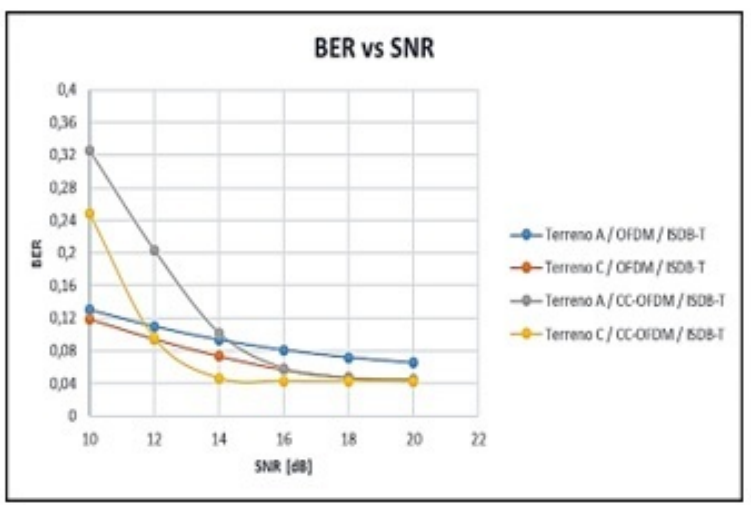

Figura 4. BER vs. SNR con imagen RGB de señal de entrada aplicando efecto multicamino para el estándar ISDB-T.
En la Tabla 4 se observa que CC-OFDM es más eficiente en ambos terrenos.

Tabla 4. BER vs. SNR con imagen RGB de señal de entrada aplicando efecto multicamino para el estándar ISDB-T

\begin{tabular}{|c|c|c|c|c|}
\hline \multicolumn{5}{|c|}{ ISDB-T } \\
\hline & \multicolumn{2}{|c|}{ OFDM VER } & \multicolumn{2}{|c|}{ CC-OFM VER } \\
\hline SNR Db & Terreno A & Terreno C & Terreno A & Terreno C \\
\hline 16 & 91,887 & 94,300 & 94,201 & 95,273 \\
\hline
\end{tabular}

A continuación, se observan los resultados obtenidos en una imagen con una variación de $\mathrm{SNR}=16[\mathrm{~dB}]$, para un terreno tipo $\mathrm{C}$ (urbano). En la Figura 5 se observa la imagen trasmitida, en la Figura 6 se observa la imagen recibida con OFDM, y en la Figura 7 la imagen recibida con CC-OFDM.

Teniendo como señal de entrada una imagen RGB se obtuvo los diferentes resultados entre BER vs. SNR en el cual se consigue resultados distintos debido a la variación que se produce en la relación señal a ruido. Esta variación se la realiza en los 2 canales SUI (Standford University Interim) implementados en la simulación con el fin de observar cuál de los estándares tiene mejor comportamiento ante este efecto multicamino como se observa en la Tabla 3. Siendo el BER menor en CC-OFDM.

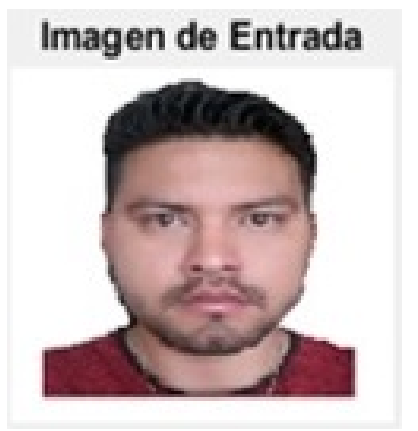

Figura 5. Imagen de entrada original.

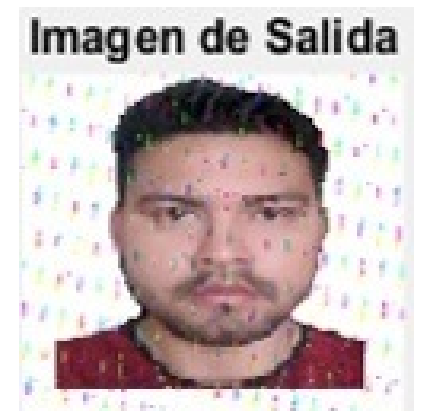

Figura 6. Imagen al pasar por el terreno $\mathrm{C}$ con $\mathrm{SNR}=16$ [dB] con modulación OFDM. 


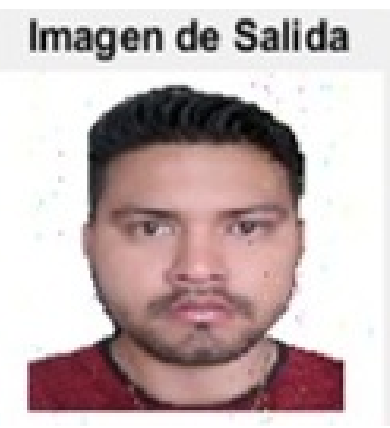

Figura 7. Imagen al pasar por el terreno $\mathrm{C}$ con $\mathrm{SNR}=16$ con modulación CC-OFDM.

\section{Conclusiones}

Para un sistema OFDM se tiene un número muy alto de portadoras para el estándar ISDB-T, ya que el ancho de banda establecido a cada portadora es reducido, disminuyendo la velocidad de símbolo de manera proporcional, aumentando el tiempo para transmitir cada símbolo, por esta razón este sistema tiene más flexibilidad a interferencias por multicamino.

Se concluyó que aplicando una modulación CCOFDM para el estándar ISDB-T es 24,95 \% más eficiente que OFDM según el BER obtenido en la simulación para un terreno urbano con un delay de 8,3640 [ms] siendo más confiable, pero con un 16,35 \% más de retardo con respecto a la modulación

\section{Referencias}

[1] M. Fuentes Muela, Evaluación de prestaciones (rendimiento e interferencias) del estándar de Televisión Digital Terrestre ISDB-Tb, mediante simulaciones y mediciones. Proyecto final de Carrera, Universidad Politécnica de Valencia, España, 2012. [Online]. Available: https://goo.gl/LQyMFm

[2] N. O. Pisciotta, "Remultiplexor isdb-tb," Memoria Investigaciones en Ingeniería, no. 12, pp. 57-69, 2014. [Online]. Available: https: //goo.gl/5UdD74

[3] —_ "Sistema isdb-tb. (primera ppart)," $U B P$ Serie Materiales de Investigación, no. 9, pp. 1-45, 2010. [Online]. Available: https://goo.gl/Tktnh9

[4] S. Hara and R. Prasad, Multicarrier Techniques for $4 G$ Mobile Communications. Norwood, MA, USA: Artech House, Inc., 2003. [Online]. Available: https://goo.gl/iLGLJj

[5] J. Armstrong, "OFDM for optical communications," Journal of Lightwave Technology, vol. 27, no. 3, pp. 189-204, Feb 2009. [Online]. Available: https://doi.org/10.1109/JLT.2008.2010061
[6] M. Engels, Wireless OFDM Systems: How to Make Them Work? Norwell, MA, USA: Kluwer Academic Publishers, 2002.

[7] V. Sharma, A. Shrivastav, A. Jain, and A. Panday, "Ber performance of ofdm-bpsk,-qpsk,-- qam over awgn channel using forward error correcting code," International Journal of Engineering Research and Applications (IJERA), vol. 2, no. 3, pp. 1619-1624, 2012. [Online]. Available: https://goo.gl/2Fs5AU

[8] M. Taguchi, K. Murayama, T. Shitomi, S. Asakura, and K. Shibuya, "Large-capacity wireless transmission technology," ITE Transactions on Media Technology and Applications (MTA), vol. 1, no. 1, pp. 34-39, 2013. [Online]. Available: https://goo.gl/XJnDxo

[9] A. Joshi and D. S. Saini, "Performance analysis of coded ofdm for various modulation schemes in 802.11a based digital broadcast applications," in Information Processing and Management, V. V. Das, R. Vijayakumar, N. C. Debnath, J. Stephen, N. Meghanathan, S. Sankaranarayanan, P. M. Thankachan, F. L. Gaol, and N. Thankachan, Eds. Berlin, Heidelberg: Springer Berlin Heidelberg, 2010, pp. 60-64. [Online]. Available: https://doi.org/10.1007/978-3-642-12214-9_11

[10] K. Thenmozhi and V. Prithiviraj, "Suitability of coded orthogonal frequency division multiplexing (cofdm) for multimedia data transmission in wireless telemedicine applications," in International Conference on Computational Intelligence and Multimedia Applications (ICCIMA 2007), vol. 4, Dec 2007, pp. 288-292. [Online]. Available: https://doi.org/10.1109/ICCIMA.2007.166

[11] M. D. Hassib, J. Mandeep, M. Abdullah, M. Ismail, R. Nordin, and M. Islam, "Improved concatenated (rs-cc) for ofdm systems," IEICE Electronics Express, vol. 9, no. 6, pp. 538-543, 2012. [Online]. Available: https://doi.org/10.1587/elex.9.538

[12] F. Tarrés and C. M., Codificación de canal II: códigos convolucionales. Universidad Oberta de Catalunya, 2012. [Online]. Available: https://goo.gl/J2WMP9

[13] S. S. Joshi and A. P. Laturkar, "Ber improvement in ofdm using coding techniques," International Journal of Electrical and Electronics Research, vol. 2, no. 3, pp. 167-173, 2014. [Online]. Available: https://goo.gl/Q22qJ2 
[14] L. R. Cantos Sánchez, S. J. Tapuy Rendón, and B. Ramos Sánchez, Simulación del estándar de Televisión Digital ISDB-Tb basado en un esquema de modulación/ Demodulación OFDM Implementado en Matlab-Simulink. Tesis de grado, Escuela Superior Politécnica del Litoral, Ecuador, 2014. [Online]. Available: https://goo.gl/mA12pc

[15] P. S. Calderón Valarezo, Análisis del desempeño de la técnica OFDM sobre canales dispersos. Tesis de grado, Universidad de las Fuerzas Armadas, Ecuador, 2007. [Online]. Available: https://goo.gl/vVu1PC

[16] J. Espitia Juárez, Codificador Reed-Solomon en software. Tesis de grado, Instituto Politécnico Nacional, México, 2012. [Online]. Available: https://goo.gl/egNKBn

[17] C. Mateos, Simulador de un sistema de Transmisión, Recepción y ecualización de DVB-T en Simulink. Tesis de grado, Universidad de Sevilla. España, s.f. [Online]. Available: https://goo.gl/WPJfWW

[18] A. Delgado Gutiérrez, Transmisión de señales de $T V$ digital en el Estándar Terreno DVB-T. Universidad Poliécnica de Madrid, España, 2002. [Online]. Available: https://goo.gl/Rwpq6N

[19] D. Argüellos and J. A., Simulación del diagrama funcional de transmisión del sistema ISDB-T, para el estudio de su estructuración. Tesis de grado, Universidad Central de Venezuela, Venezuela., 2017. [Online]. Available: https://goo.gl/tu83My

[20] N. Pérez, Gestión del espectro radioeléctrico para $T V$ digital. Universidad de los Andes, Venezuela., 2013.
[21] S. Landeros-Ayala, S. A. Chávez-Cárdenas, and J. C. González-Sánchez, "Análisis de la eficiencia de los estándares de transmisión de televisión digital." Ingeniería Investigación y Tecnología, vol. 14, no. 3, pp. 335-353, 2013. [Online]. Available: https://goo.gl/sw7jxt

[22] DIBEG. (2018) Comparison of 3 DTTB systems. Digital Broadcasting Experts Group. [Online]. Available: https://goo.gl/rJomDY

[23] CRC, Definición de las especificaciones técnicas de la TDT en Colombia. Comisión de Regulación de Comunicaciones, Colombia., 2012. [Online]. Available: https://goo.gl/A5iy6T

[24] R. G. Acosta Arias, Estudio teórico práctico de los códigos no binario de Reeds-Solomon. Tesis de grado, Escuela Politécnica Nacional, Ecuador., 1994. [Online]. Available: https://goo.gl/nMohzK

[25] MathWorks. (2018) Communications toolbox - examples. The MathWorks, Inc. [Online]. Available: https://goo.gl/RY85fW

[26] A. F. Padilla Narváez, Detección y corrección de errores en códigos convolucionales mediante el algoritmo de Viterbi usando una microcomputadora. Tesis de grado, Escuela Politécnica Nacional, Ecuador, 1985. [Online]. Available: https://goo.gl/9nLXYm

[27] L. Arnone, C. Gayoso, C. González, J. C. García, and J. Castiñeira, Diseño de un decodificador viterbi para ser utilizado en enlaces infrarrojos. Universidad Nacional del Mar del Plata, 2012. [Online]. Available: https://goo.gl/gK3bMk 